HEALING ARTS: MATERIA MEDICA

\title{
Materia Medica Submission: Six Lessons from a Previous Pandemic
}

\author{
Eileen Barrett, MD, MPH, SFHM, MACP® \\ Division of Hospital Medicine, Department of Internal Medicine, MSC 10-5550, University of New Mexico, Albuquerque, NM, USA.
}

$\mathrm{J}$ Gen Intern Med 36(1):222-3

DOI: $10.1007 / \mathrm{s} 11606-020-06300-4$

(c) Society of General Internal Medicine 2020

W hen there were dire shortages of Ebola workers during the 2014 pandemic, I applied to volunteer and was accepted after two applications and interviews. Once deployed, the training included supportive care basics, PPE protocols, cultural humility, and self-care. The context of care was foreign, however, and felt like a betrayal of our core principles: if there were unsafe conditions then the patients would not receive care from us until we could be safe providing it. This was deeply unsettling at best, and horrifying at worst. How could we not attend to them when sick? Our trainer patiently and compassionately explained that we needed to take care of ourselves so we could care for others - offering the first of several leadership lessons that changed my thinking and my practice, and fundamentally changed me.

Providing care during Ebola is difficult to describe to most people because they can't have a context for the work. In full PPE and in triple digit temperatures with dense humidity, we hand carried basic supplies including reconstituted oral rehydration salts, linens, medications, and bags of IV fluids, then hung those same fluids and antibiotics, changed linens, and encouraged patients to eat or drink. Labs could only be performed early or late in the day due to equipment malfunction from heat, there were power outages daily, the air was pungent from open burning of medical waste, and the tap water wasn't potable. Patients often died alone, on their mattresses directly on the floor, because loved ones could not visit them. Touchingly, infectious patients who were strong enough provided care to the sickest—reminding me that patients are always the most important members of our teams and the reason why we are here.

The first time I was splattered with infectious fluids, I was so focused on the environment around me that I didn't recognize what had happened. After providing the patient a clean

Received April 24, 2020

Accepted October 6, 2020

Published online November 2, 2020 sheet and my colleague sterilizing my gloves, apron, suit, and boots, we couldn't disinfect my face shield because of the risk of getting bleach in my eyes. When completing our work and getting to the doffing station, I was burning to get my face shield off. Once it was off, however, I realized that despite spending the last hour longing for cool air on my sweatsaturated scrubs and hair, that I was terrified of removing my plastic suit, gloves, and N95. After what felt like ten minutes but was likely 30 seconds, I collected myself and carefully doffed my remaining PPE with my designated doffing buddy coaching me-reminding me that we do our best work when we do it together.

Soon afterward, community-based infection control measures were more effective and the number of new cases fell. I was asked to go to a local hospital devastated by Ebola, where two months earlier patients had been locked inside a fence without any clinicians and had food and water thrown over the gate to them. These circumstances were drastically improved by the time of my arrival, but the ongoing devastation shocked and overwhelmed me. Patients were lying in urine and stool with bedsores and were often undertreated due to supply and staff shortages. Ebola screening was sporadic so staff and patients were afraid as we grieved while providing the best care we could.

After one particularly difficult day, I started to cry and cried during dinner, in the shower, and in my tent, and what started as tears turned into full body sobs that I feared might not stop. But the silent, kind, knowing nods of friends and supportive and loving words from my husband six time zones away were immeasurably soothing and healed me enough so I could eventually sleep and return to work in the morning. I came to love the work for my admiration of my brave colleagues, friends, and patients, as well as the great sense of purpose that came from the privilege of being able to be a doctor with them and for them-a lesson that we are strengthened from working with a shared sense of purpose. At the same time, I realized that there would be no end to my grief.

We often describe experiences as life changing, but of course they are only life changing if they truly change our lives. Ebola work changed me by helping small gestures of kindness move me to tears, making me aware of the consequences that the accident of my birth was in a wealthy country, and making me more generous with those who don't yet see their many privileges - just as I sometimes do and sometimes don't yet. It also made me impatient and at times seethe at 
processes and systems that keep us from providing care people deserve - teaching me that every one of us has a moral duty in undoing these in big and small ways, and that we can start to heal when we do so.

The pandemic humbled me and reminded me that structural inequity and racism are pervasive and devastating, robust public health systems save lives, and we need each other more than any of us can say. It was heartbreaking, difficult, and treacherous work, but it was also beautiful and glorious because of the humanity of others - just like the practice of medicine everywhere, everyday. I volunteered then and would do so again, first and foremost to serve the patients-but closely following that is so I would again be so moved by the compassionate heroism and heroic compassion of the patients and my colleagues. And I thank you for doing the same right now during this pandemic - reminding me of a sixth lesson, to thank sincerely and often. Thank you for being a COVID physician, and please know doing so will change you in ways you may not anticipate, in many ways for the better. Your strength will come from the kindness you receive, the grief you feel, the colleagues who strengthen you, and the patients who deserve you.

Corresponding Author: Eileen Barrett, MD, MPH, SFHM, MACP; Division of Hospital Medicine, Department of Internal Medicine, MSC 10-5550, University of New Mexico, Albuquerque, NM, USA (e-mail: ebarrett@salud.unm.edu).

Publisher's Note Springer Nature remains neutral with regard to jurisdictional claims in published maps and institutional affiliations. 